\title{
Targeting PI3K/Akt represses Hypoxia inducible factor-1 a activation and sensitizes Rhabdomyosarcoma and Ewing's sarcoma cells for apoptosis
}

Mehtap Kilic-Eren ${ }^{1 *}$, Tulin Boylu² and Vedrana Tabor ${ }^{3}$

\begin{abstract}
Background: Hypoxia inducible factor-1 a (HIF-1a) has been identified as an important novel target in apoptosis resistance of pediatric tumors such as Rhabdomyosarcoma (RMS) and Ewing's sarcoma (ES). Evidence suggests that PI3K/Akt signaling plays a role in regulation of HIF-1a activation as well as apoptosis resistance in various adult tumors. However the relevance of PI3K/Akt signaling in HIF-1 ba activation and apoptosis resistance in childhood tumors has not been addressed yet. Thus, this study was to investigate whether PI3K/Akt signaling is involved in hypoxia induced activation of HIF-1a as well as in resistance to hypoxia-induced apoptosis in childhood tumors such as RMS and ES.
\end{abstract}

Methods: Constitutive activation of PI3K/Akt signaling was analyzed by Western blotting. Hypoxic activation of HIF-1a was determined by Western Blot analysis and electrophoretic mobility shift assay. Apoptosis was determined by flow cytometric analysis of the propidium iodine stained nuclei of cells treated with PI3K inhibitor LY294002 in combination with either TNF-related apoptosis-inducing ligand (TRAIL) or doxorubicin.

Results: This study demonstrated that PI3K/Akt signaling was constitutively activated in RMS and ES cell lines, A204 and A673, respectively. Targeting PI3K/Akt signaling by the inhibitor LY294002 (30 $\mu \mathrm{M})$ significantly decreased the protein expression as well as DNA binding activity of HIF-1a and restored the apoptosis-inducing ability of cells in hypoxia Additionally, pretreatment with LY294002 sensitized A204 and A673 cells to TRAlL or doxorubicin induced apoptosis under hypoxia.

Conclusion: These results suggest that the constitutively active PI3K/Akt signaling contributes to hypoxic activation of HIF-1a as well as HIF1a-mediated apoptosis resistance in RMS and ES cells under hypoxia.

Keywords: PI3K/Akt, Hypoxia, HIF-1a, Apoptosis, Rhabdomyosarcoma, Ewing's sarcoma

\section{Background}

Hypoxia inducible factor-1 (HIF-1) is the major transcription factor activated to mediate adoptive responses under hypoxia [1]. HIF-1 is a heterodimeric protein composed of oxygen regulated $\alpha$ and constitutively active $\beta$ subunits. When oxygen is present, HIF- $1 \alpha$ is hydroxylated by prolylhydroxylases that allows its interaction with von Hippel Lindau (VHL) complex, leading to

\footnotetext{
* Correspondence: mkiliceren@adu.edu.tr

${ }^{1}$ Department of Medical Biology, Faculty of Medicine, Adnan Menderes

University, Aydin, Turkey

Full list of author information is available at the end of the article
}

its ubiquitination and proteosomal degradation. In contrast, when oxygen is not available rate of asparagine and proline hydroxylation decreases and HIF- $1 \alpha$ cannot bind to VHL complex and remains stabilized. Stabilized HIF- $1 \alpha$ translocates to the nucleus to interact with the coactivators HIF-1 $\beta$ and $\mathrm{p} 300 / \mathrm{CBP}$ which results in transcriptional activation of the various genes including growth factors, angiogenic factors, anti-apoptotic factors and the factors involved in anaerobic metabolism $[2,3]$. HIF- $1 \alpha$ is overexpressed in a variety of human tumors associated with poor prognosis and resistance to chemotherapy-induced apoptosis [4]. In our previous 
work we also identified HIF-1 $\alpha$ as an important target modulating apoptosis resistance in pediatric tumors such as Rhabdomyosarcoma (RMS) and Ewing's sarcoma (ES) [2]. Constitutive activation of phosphatidylinositol 3-kinase (PI3K), due to a variety of genetic aberrations, is frequently observed in human cancers and plays a major role in tumor formation and progression [5,6]. Akt, a serine/threoneine kinase, is a central mediator of the PI3K with several downstream targets. Aberrant activation of PI3K/Akt plays important role in the resistance of tumor cells to anticancer therapy $[7,8]$. Emerging evidences suggest that PI3K/Akt signaling mediates regulation and activation of HIF- $1 \alpha$ in various human cancers [9-11]. However, to date there is no data signifying the relevance of PI3K/Akt signaling in activation of HIF- $1 \alpha$ and in resistance to apoptosis under hypoxia in childhood tumors.

RMS is the most common soft tissue sarcoma in children and accounts for $23 \%$ of all sarcomas, and $7 \%$ of all pediatric malignancies $[2,12]$. ES is the second most common primary malignant bone tumor $[2,13]$. Although the majority of RMS and ES patients with non-metastatic disease can be cured, the prognosis of patients with metastatic disease remains inferior $[14,15]$. Therefore, it is of critical importance to understand the key factors and molecular pathways in pathogenesis and survival of RMS and ES in order to develop novel effective anticancer strategy. Published data indicates that the increased levels of phosphorylated, hence active, Akt in childhood cancer samples, including neuroblastoma, glioblastoma, RMS and ES, is negatively correlated with patient survival [16-20]. Accordingly, this study was undertaken to investigate whether constitutive PI3K/Akt signaling is involved in regulation of HIF- $1 \alpha$ activation as well as resistance to hypoxia-induced apoptosis in human RMS and ES cell lines A204 and A673, respectively.

\section{Results \\ PI3K/Akt signaling is constitutively activated in A204 RMS and A673 ES cells}

To assess the role of PI3K pathway in HIF- $1 \alpha$ induction the phosphorylation status of Akt, which was used as surrogate for PI3K activity, was examined by Western blot analysis. As shown in Figure 1A and B, A204 and A673 cells had high levels of Akt phosphorylation on Ser473 in normoxia. Next we used LY294002, the pharmacologic inhibitor of PI3K to interfere with phosphorylation of Akt, and here we show that upon treatment with LY294002 the level of p-Akt was decreased in a dose dependent manner in both A204 and A673 cells in normoxia (Figure 1A, B). To address whether PI3K/ Akt signaling was sustained in hypoxia, phosphorylation of Akt was examined in the presence or absence of LY294002 in both normoxia and hypoxia. In addition, due to the growth factors present in serum, which can induce Akt phosphorylation, we also tested serumdeprived cells. Accordingly, pretreatment of A204 and A673 cells by $30 \mu \mathrm{M}$ LY294002 decreased phosphorylation of Akt in both conditions whereas protein levels of total Akt were not altered (Figure 1C, D). As seen in Figure $1 \mathrm{C}$ and D, levels of p-Akt-Ser473 were similar in A204 and A673 cells either in normoxia or hypoxia and did not change by serum deprivation but suppressed by LY294002 addition. Densitometry analysis also confirmed these data (Figure 1E and F) suggesting in A204 and A673 cells in normoxia p-Akt levels when normalized to Akt levels, is significantly decreased in the presence of LY294002 whether or not FCS is withdrawn. In contrast, no significant differences were detected in p-Akt levels between hypoxia and normoxia in both cells. In hypoxia in A673 cells p-Akt levels again did not change by serum deprivation whereas it seemed to be increased in A204 cells though it was not significant. Moreover, addition of LY294002 was able to suppress p-Akt levels significantly either in the presence or absence of FCS also in hypoxia in both cells. Whereas pretreatment of A204 and A673 cells by $30 \mu \mathrm{M}$ LY294002 did not alter the protein levels of total Akt. Hence, we conclude that levels of $\mathrm{p}$-Akt were sustained in both cells under hypoxia and did not change by serum deprivation either in normoxia or hypoxia.

Thus, these data demonstrated that activation of PI3K/ Akt signaling is constitutive in both cell lines in normoxia and hypoxia, as evidenced by high levels of phosphorylated p-Akt-Ser473, the downstream effector of PI3K (Figure 1C, D).

\section{PI3-K/Akt signaling is involved in hypoxic induction of HIF-1 alpha protein and DNA binding activity in A204 and A673 cells}

In order to examine whether constitutive activation of PI3K/Akt signaling is involved in hypoxic induction of HIF- $1 \alpha$ protein, either pretreated with $30 \mu \mathrm{M}$ of LY294002, or left untreated (control) A204 and A673 cells were incubated under hypoxic conditions and subsequently subjected to Western blot analysis for stabilization of HIF-1 $\alpha$ protein. As shown in Figure 2A, HIF- $1 \alpha$ protein was stabilized $24 \mathrm{~h}$ after exposure to hypoxia and remained its levels up to $48 \mathrm{~h}$ post exposure in both cell lines. Remarkably, pre-treatment with LY294002 decreased the expression of HIF- $1 \alpha$ suggesting that induction of HIF-1 $\alpha$ by hypoxia requires activation of PI3K pathway. Further, the effect of PI3K inhibitor on hypoxia-induced DNA-binding activity of HIF- $1 \alpha$ was investigated by EMSA utilizing a $3^{\prime}$ HRE-derived oligonucleotide probe. Using a mutant probe, performing competition assays (Figure 2B) confirmed the identity of the HIF- $1 \alpha$ band. Under hypoxic conditions HIF- $1 \alpha$ showed increased DNA-binding activity at $24 \mathrm{~h}$ and the 


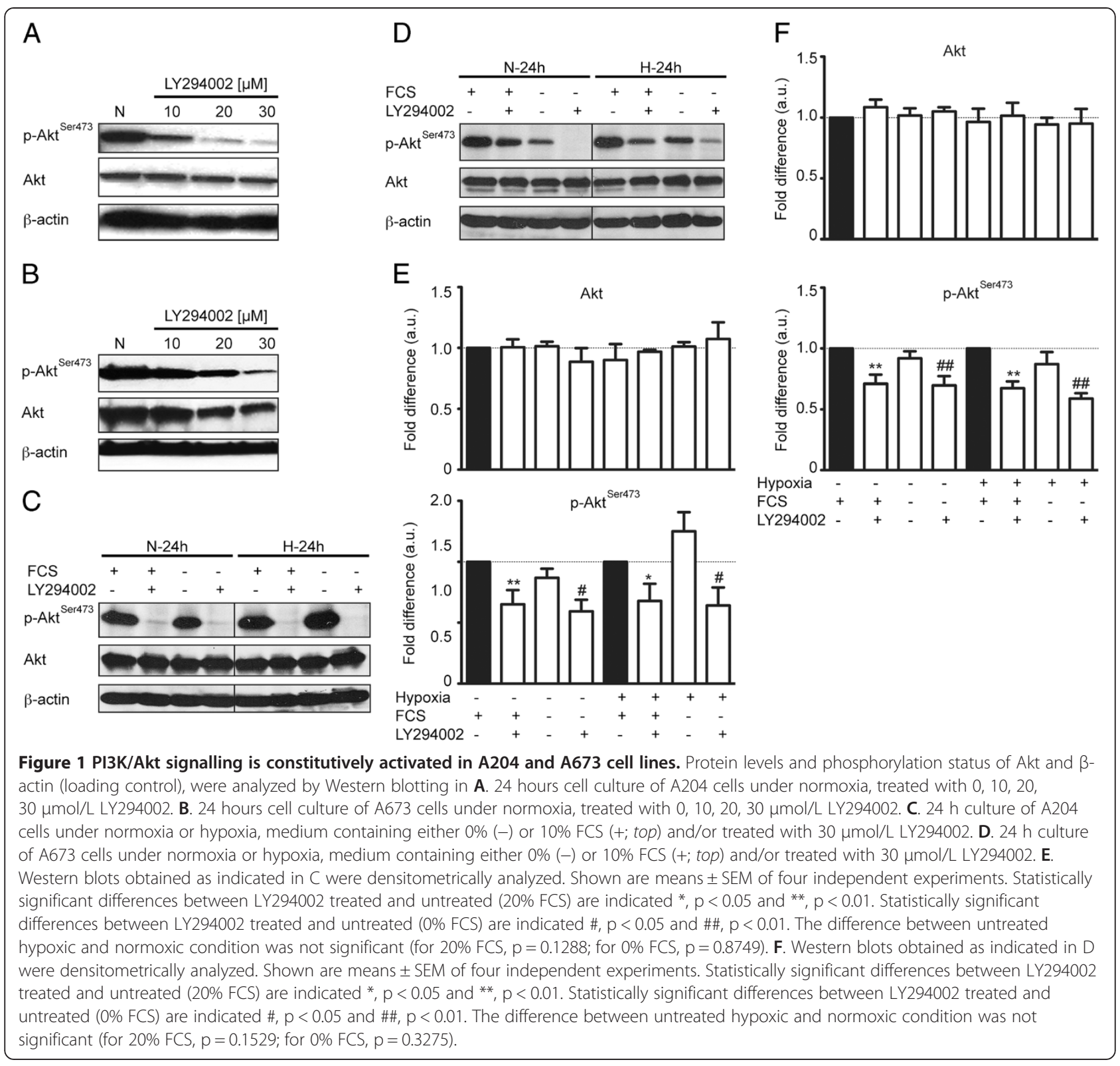

level of activity was still high at $48 \mathrm{~h}$ in both A204 and A673 cells (Figure 2B). Pretreatment with LY294002 reduced hypoxia-induced DNA binding activity of HIF-1 $\alpha$ in both cell lines, although this effect was more pronounced in A204 cells after $24 \mathrm{~h}$ compared to A673 cells.

\section{Inhibition of HIF-1a by LY294002 restores apoptosis inducing ability of A204 and A673 cells under hypoxia}

Next, we investigated whether or not decreased stabilization and DNA binding activity of HIF- $1 \alpha$ by LY294002, can sensitize A204 and A673 cells to apoptosis under hypoxia. In order to examine long lasting effects of LY294002, cells were cultured for up to $72 \mathrm{~h}$ under hypoxic conditions in the presence of $30 \mu \mathrm{M}$
LY294002 and apoptosis was assessed every 24 hours. As seen in Figure 3A while hypoxia alone did not trigger apoptosis in both cell lines, pretreatment with LY294002 induced $15,5( \pm 1,7) \%$ and $16,0 \quad( \pm 1,1) \%$ apoptosis in A204 and A673 cells, respectively, in a time dependent manner (Figure 3A). These data suggest that decreased protein level and DNA binding activity of HIF- $1 \alpha$ by LY294002 treatment restores apoptosis sensitivity in A204 RMS and A673 ES cells.

In our previous work, we showed that hypoxia protects against death receptor- (e.g. TRAIL) and cytotoxic drug- (e.g. Doxorubicin) induced apoptosis in A204 and A673 cells. Therefore cells were pretreated with LY294002 and cultured for up to $72 \mathrm{~h}$ in the presence or 


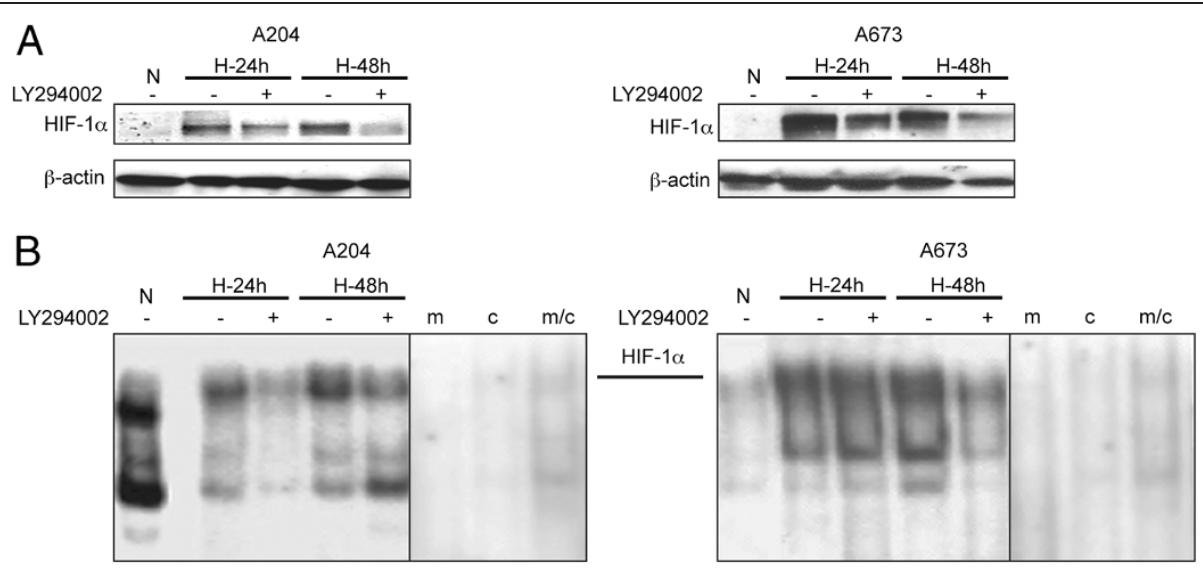

Figure 2 PI3K/Akt pathway is involved in activation of HIF-1a in A204 and A673 cell lines. Cells were pretreated with 0 or $30 \mu \mathrm{mol} / \mathrm{L}$ of LY294002 for 1 hour and then incubated for 24 hours or 48 hours either in normoxia (N) or hypoxia (H). A. Protein expression levels of HIF-1a and $\beta$-actin were analyzed by western blotting. B. DNA binding activity of Hif-1a in nuclear extracts was assessed by EMSA. Specific HIF-1 DNA binding was confirmed by using a radioactive labeled mutated $(\mathrm{m})$ probe, in which the HIF-1a consensus binding site is inactivated, and by competition with unlabelled consensus (c) and mutant (c/m) DNA probes in 100 fold excess.
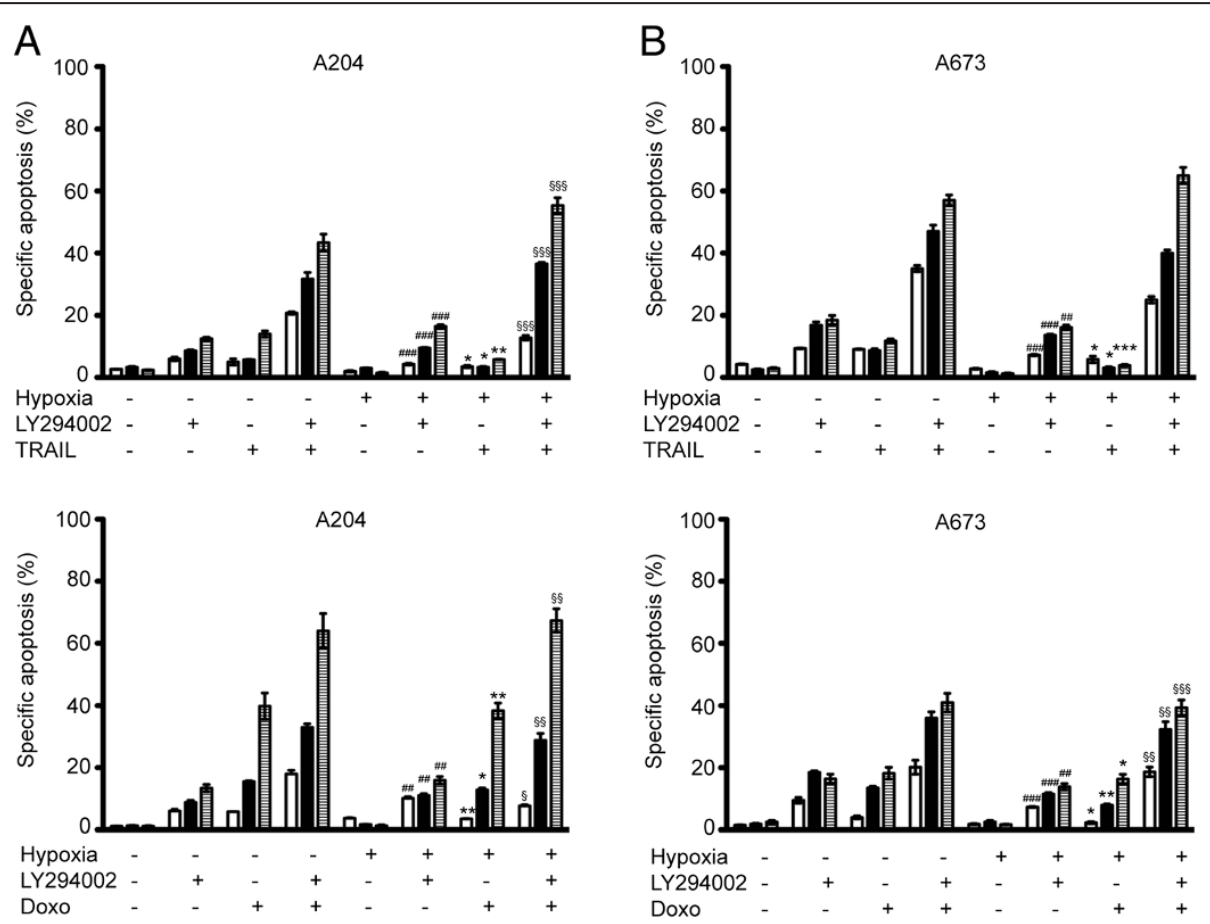

Figure 3 Sensitization of A204 and A673 cells to doxorubicin- and TRAIL-induced apoptosis by PI3K inhibition. A204 (A) and A673 (B) cells were pretreated or not with $30 \mu \mathrm{mol} / \mathrm{L}$ LY294002 for 1 hour, incubated with doxorubicin $(0.1 \mu \mathrm{g} / \mathrm{ml})$ or TRAlL $(100 \mathrm{ng} / \mathrm{ml})$ and cultured under normoxic or hypoxic conditions for up to 72 hours. 24 hours-white bars, 48 hours- black bars, 72 hours-hatched bars. Apoptosis was determined by FACS analysis of DNA fragmentation of propidium iodide-stained nuclei; the percentage of specific apoptosis is shown. Columns, mean of three independent experiments done in duplicate; bars, SD. For statistical analysis two-way ANOVA was performed comparing specific apoptosis of either TRAIL or doxorubicin-induced apoptosis under normoxia vs hypoxia $\left({ }^{*} p<0.05,{ }^{* *} \mathrm{p}<0.01,{ }^{* *} \mathrm{p}<0.001\right)$ and hypoxia induced apoptosis vs hypoxia + LY294002 (\#\# $\mathrm{p}<0.01, \# \# \mathrm{p}<0.001)$ as well as hypoxia + TRAlL or doxorubicin vs hypoxia + LY294002 + TRAlL or doxorubicin $\left({ }^{\S \S} p<0.01,{ }^{\S \S} p<0.001\right)$. 
absence of TRAIL in both normoxia and hypoxia. Apoptosis was assessed every 24 hours, and as seen in Figure 3A, without LY294002 pretreatment, after $72 \mathrm{~h}$ TRAIL - induced apoptosis in normoxia was at least $10 \%$ higher than to that of in hypoxia, underlining the protective role of hypoxia in both cell lines. Interestingly, pretreatment with LY294002 significantly sensitized cells for TRAIL-induced apoptosis and rendered the protective effect of hypoxia (Figure 3A).

Next, the effect of HIF-1 $\alpha$ inhibition by LY294002 treatment in combination with doxorubicin, typically triggering apoptosis via the mitochondrial pathway, was also tested. In contrast to TRAIL, doxorubicin-induced apoptosis was substantial in A204 and A673 cells under either normoxia or hypoxia, though a slight protective effect of hypoxia was still present. Pretreatment of cells with LY294002 greatly enhanced doxorubicin-induced apoptosis. When pretreated with LY294002 the rate of apoptosis was at least 20\% higher in both A204 and A673 cells after $72 \mathrm{~h}$ exposure to hypoxia (Figure 3B).

Moreover, the broad range caspase inhibitor z-VAD-fmk was used to test requirement for caspases during TRAILor doxorubicin-induced apoptosis under hypoxia. Apoptosis induced by combined treatments of LY294002 and TRAIL, or doxorubicin was significantly blocked in the presence of z-VAD-fmk under both normoxia and hypoxia in both cell lines in a time dependent manner (Figure 4A and B). These results indicated that apoptosis induced by combined treatments with LY294002 and either TRAIL, or doxorubicin was mediated by caspases.

\section{Discussion}

Previously, HIF-1 $\alpha$ has been identified as key factor in conferring resistance to apoptosis under hypoxia in childhood tumors such as RMS and ES [2]. Evidences suggest that PI3K/Akt signaling plays a role in regulation of HIF- $1 \alpha$ activation in various adult tumors [8,21-25]. The present study was undertaken to investigate the relevance for PI3K/Akt signaling and HIF-1 $\alpha$ activation along with apoptosis resistance in RMS and ES. Here, it is presented for the first time that constitutively activated PI3K/Akt involved in hypoxic activation of HIF- $1 \alpha$ and targeting PI3K/Akt via LY294002 prevented HIF-1 $\alpha$ 's stabilization and restored apoptosis sensitivity of RMS and ES cells under hypoxic conditions.

The current study provides a number of evidences supporting this conclusion. First, we demonstrated that A204 (RMS) and A673 (ES) cell lines show high level of phosphorylated Akt (on Ser473), sustaining under serum deprivation and hypoxia indicating to the constitutive activation of Akt. Our data is consistent with the in vivo studies, showing the increased abundance of active, phosphorylated, Akt in several childhood cancers, including RMS and ES [16-20,26]. Second, Akt phosphorylation was inhibited via PI3K inhibitor LY294002 that also decreased the protein expression and DNA binding activity of HIF-1 $\alpha$. More importantly inhibition of PI3K/ Akt signaling or HIF-1 $\alpha$ activity by LY294002 blocked protection against hypoxia-induced cell apoptosis. Third, inhibition of HIF-1 $\alpha$ activation via LY294002 also sensitized RMS and ES cells for death receptor (TRAIL) - as well as drug (Doxorubicin) - induced apoptosis which could be blocked in the presence of z.VAD. fmk.

Oxygen regulated transcription factor HIF-1 $\alpha$ and the serine/threonine kinase Akt are both essential for development and implicated in tumor growth [8,27-31]. They share the ability to induce processes such as angiogenesis, glucose uptake, and glycolysis [29,32-34]. To date several studies have identified the PI3K/AKT pathway as an important element in hypoxic induction of HIF- $1 \alpha$ protein and activity in tumor cell lines [9,11,35,36] Also, in non malignant systems such as developing rat brain or pulmonary artery smooth muscle cells PI3K/Akt pathway is involved in activation of HIF-1 $\alpha[21,37,38]$.

From our data, we propose that constitutive activation of the PI3K/Akt contributes to the increased hypoxic activation of HIF- $1 \alpha$ in RMS and ES cells, because inhibiting PI3K/Akt activity by the inhibitor LY294002 decreased HIF-1 $\alpha$ protein levels and prevented DNA binding activity under hypoxia. However, there are other reports indicating the contrary data and suggesting that PI3K/Akt signaling is neither required nor sufficient for the hypoxic stabilization or activation of HIF-1 $\alpha[39,40]$. Hence, one possibility is that the involvement of constitutive PI3K/Akt signaling in hypoxic activation of HIF- $1 \alpha$ may depend on cell type or on tumor type/stage and its microenvironment.

The PI3K/Akt pathway is also well known to mediate prosurvival signals. In particular, Akt is involved in inhibition of apoptosis by phosphorylating pro-apoptotic molecules i.e. Bad, Caspase-9 or modulating transcription factors i.e. c-Raf. [41]. Recent studies have shown that inhibition of PI3K/Akt might be a promising strategy to decrease the threshold for apoptosis induction via the death receptor triggering or cytotoxic drugs in neuroblastoma and glioblastoma $[8,26,42]$. In line with that our data also provides evidence that PI3K/Akt inhibition cooperates with TRAIL or doxorubicin to trigger apoptosis under hypoxia in RMS or ES cells. Resistance to apoptosis is still major obstacle in treatment and our findings may have important implication for apoptosis-based therapy of RMS and ES. Moreover it provides basis for further investigation of new generation PI3K inhibitors in combination with TRAIL or chemotherapy to overcome apoptosis resistance associated with tumor hypoxia. Similarly a previous report also suggests 3-phosphoinositide-dependant kinase-1(PDK-1)/Akt pathway as an attractive therapeutic target in RMS [17]. 

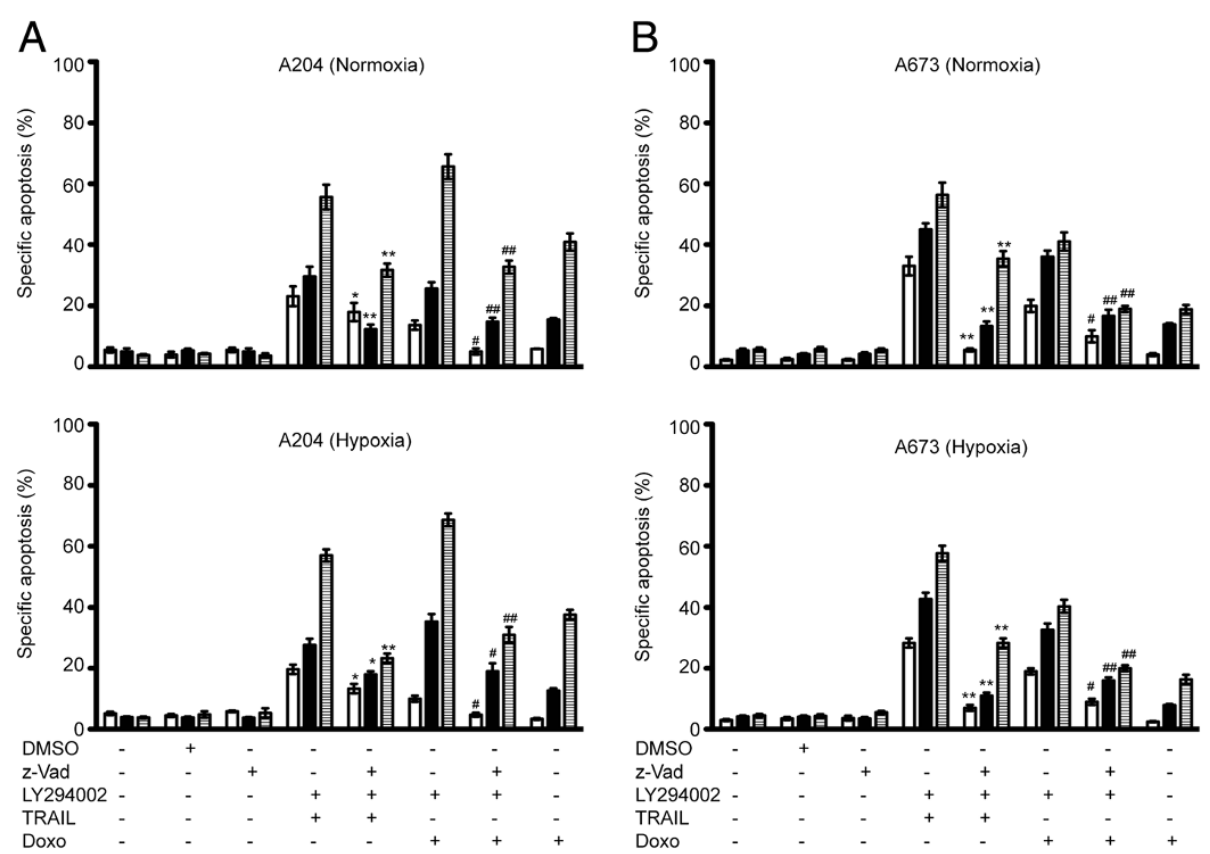

Figure 4 Sensitization of A204 and A673 cells for doxorubicin- and TRAIL- induced apoptosis is caspase dependent. A204 (A) and A673 (B) cells were pretreated with 0 or $30 \mu \mathrm{mol} / \mathrm{L}$ of LY294002 for 1 hour, and cultured under normoxic or hypoxic conditions with doxorubicin

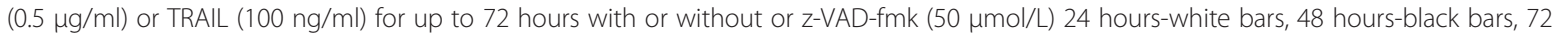
hours-hatched bars Columns, mean of three independent experiments done in duplicate; bars, SD. For statistical analysis two-way ANOVA was performed comparing specific apoptosis of either LY294002 + TRAIL or doxorubicin--induced apoptosis without z-VAD-fmk vs with z-VAD-fmk under normoxia ( $\left.{ }^{*} p<0.05,{ }^{* *} p<0.01\right)$ or the same comparison of specific apoptosis under hypoxia $\left({ }^{\#} p<0.05,{ }^{\# \#} p<0.01\right)$.

It will be the object of our further investigations to elucidate the exact role of PI3K/Akt in hypoxic activation of HIF- $1 \alpha$ and to identify the molecules mediating the sensitization effect of PI3K/Akt.

\section{Conclusion}

Constitutive activation of PI3K/Akt involved in hypoxic activation of HIF- $1 \alpha$ in RMS and ES cells. Targeting PI3K/Akt via LY294002 prevented HIF-1 $\alpha$ 's stabilization and restored apoptosis sensitivity of RMS and ES cells under hypoxic conditions. The current study identifies an important link between PI3K/AKT and HIF-1 $\alpha$, which may have particular relevance to disease progression as well as therapeutic target for cancer intervention in RMS and ES.

\section{Materials and methods}

\section{Cell Culture and Hypoxia incubation}

Human Rhabdomyosarcoma (A204) and Ewing's sarcoma (A673) cell lines were obtained from American Type Culture Collection (Manassas, VA) and were grown in Dulbecco's modified Eagle's medium (Life Technologies, Inc., Eggenstein, Germany) containing 10\% heat inactivated fetal calf serum (Biochrom, Berlin, Germany), $100 \mathrm{IU} / \mathrm{ml}$ penicillin, $100 \mu \mathrm{g} / \mathrm{ml}$ streptomycin (Biochrom), $10 \mathrm{mM}$ glutamine (Biochrom) in a humidified atmosphere at $37{ }^{\circ} \mathrm{C}$ with $5 \% \mathrm{CO}_{2}$ unless otherwise specified. Hypoxic conditions $\left(0.5 \% \mathrm{O}_{2}\right)$ were achieved by incubation in a humidified internal incubator of a hypoxia glove box (Coy Laboratory Products, Inc.). After an initial exposure to low oxygen, all subsequent treatments were given within the glove box to prevent cellular damage due to reoxygenation.

\section{Determination of apoptosis}

Apoptosis was assessed by fluorescence-activated cellsorting (FACScan, Becton Dickinson, Heidelberg, Germany) analysis of DNA fragmentation of propidium-iodide stained nuclei as described previously [2]. The percentage of specific apoptosis was calculated as follows: $100 \times$ [experimental apoptosis (\%) - spontaneous apoptosis (\%)] [100\% spontaneous apoptosis (\%)].

\section{Protein extraction and Western blot analysis}

Total cell extracts were prepared from cells grown in 6-well plates at $90 \%$ confluence. Cells were exposed to $20 \% \mathrm{O}_{2}$ or $0.5 \% \mathrm{O}_{2}$ for the indicated time points and lysed in lysis buffer (20 mM Tris, pH 7.5 (Sigma), $150 \mathrm{mM} \mathrm{KCl} \mathrm{(Sigma),}$ $1 \mathrm{mM}$ EDTA, 1\% Triton X-100 (Sigma) supplemented with protease inhibitor mixture (Complete ${ }^{\circledR}$; Roche Applied Science, Mannheim, Germany). $0.2 \mathrm{mM}$ phenylmethylsulfonyl fluoride (PMSF); $0.5 \mathrm{mM}$ dithiothreitol (DTT) and $1 \mathrm{mM}$ sodium-ortho-vanadate before use. Western blot analysis 
was done as described previously using primary antibodies, mouse anti-Hif-1 $\alpha$ monoclonal (1:250; BD Biosciences; Heidelberg, Germany), rabbit anti-phospho Akt (Ser 473) (D9E) and rabbit anti-Akt (Cell Signaling, Beverly, MA), followed by goat-anti-mouse IgG or goat-anti-rabbit IgG conjugated to horseradish peroxidase (1:5,000; Santa Cruz Biotechnology) [2]. Mouse anti- $\beta$-actin monoclonal antibody (1: 5000; Sigma), was used as a loading control. All proteins were visualized using enhanced chemiluminescence (Amersham Biosciences).

\section{Nuclear protein extraction and Electrophoretic mobility shift assay (EMSA)}

Nuclear extracts were prepared essentially as described [2]. Oligonucleotide DNA probes containing the HIF-1 $\alpha$ binding sequence 5'-TCTGTACGTGACCACACTCAC CTC [43] and a mutant probe $5^{\prime}$-TCTGTAAAAGA CCACACTCACCTC, labelled with with $\gamma$-32P-ATP (Amersham, Freiburg, Germany) and annealed with complementary oligonucleotides, were used for EMSA [43]. EMSA was done as described previously [2]. In general, unless otherwise stated, all qualitative analyses were repeated at least three times.

\section{Abbreviations}

PI3K: Phosphatidylinositol 3-kinase; Akt: Protein kinase B (PKB); HIF1a: Hypoxia inducible factor 1 alpha; HIF1ß: Hypoxia inducible factor 1 beta;

RMS: Rhabdomyosarcoma; ES: Ewing's sarcoma; TRAIL: TNF-related apoptosisinducing ligand; VHL: Von Hippel Lindau; CBP: CREB binding protein; EMSA: Electrophoretic mobility shift assay; PDK: 1-3-phosphoinositidedependant kinase-1.

\section{Competing interests}

"The authors have declared that no competing interest exists".

\section{Authors' contribution}

MKE was involved in the planning and supervision of the project, funding acquisition from received grant, conducting the experiments, data analyses and interpretation, writing and revision of the manuscript. $\mathrm{TB}$ and $\mathrm{VT}$ were involved in conducting experiments, data analyses and interpretation and drafted the article. All authors have contributed and approved the final manuscript.

\section{Acknowledgements}

This work is supported by Adnan Menderes University Scientific Research Foundation and partly by TUBITAK grant to M.Kilic-Eren, (TPF-12033) and (10853789), respectively.

\section{Author details}

${ }^{1}$ Department of Medical Biology, Faculty of Medicine, Adnan Menderes University, Aydin, Turkey. ${ }^{2}$ Department of Histology and Embryology, Faculty of Medicine, Adnan Menderes University, Aydin, Turkey. ${ }^{3}$ Present address: Department of Medical Biochemistry and Biophysics, Karolinska Institute, Stockholm, Sweden.

Received: 14 November 2012 Accepted: 10 April 2013

Published: 16 April 2013

\section{References}

1. Semenza GL: Oxygen homeostasis. Wiley Interdiscip Rev Syst Biol Med 2010, 2(3):336-361.

2. Kilic M, Kasperczyk H, Fulda S, Debatin KM: Role of hypoxia inducible factor-1 alpha in modulation of apoptosis resistance. Oncogene 2007, 26(14):2027-2038.
3. Semenza GL: Hypoxia-inducible factor 1: master regulator of $\mathrm{O} 2$ homeostasis. Curr Opin Genet Dev 1998, 8(5):588-594.

4. Semenza GL: Targeting HIF-1 for cancer therapy. Nat Rev Cancer 2003, 3(10):721-732

5. Engelman JA: Targeting PI3K signalling in cancer: opportunities, challenges and limitations. Nat Rev Cancer 2009, 9(8):550-562.

6. Sheppard K, Kinross KM, Solomon B, Pearson RB, Phillips WA: Targeting PI3 Kinase/AKT/mTOR Signaling in Cancer. Crit Rev Oncog 2012, 17(1):69-95.

7. Chen $Y$, Wang $B C$, Xiao Y: PI3K: A potential therapeutic target for cancer. J Cell Physiol 2012, 227(7):2818-2821.

8. Fulda S: The PI3K/Akt/mTOR pathway as therapeutic target in neuroblastoma. Curr Cancer Drug Targets 2009, 9(6):729-737.

9. Zhong H, Chiles K, Feldser D, Laughner E, Hanrahan C, Georgescu MM, Simons JW, Semenza GL: Modulation of hypoxia-inducible factor 1alpha expression by the epidermal growth factor/phosphatidylinositol 3-kinase/PTEN/AKT/ FRAP pathway in human prostate cancer cells: implications for tumor angiogenesis and therapeutics. Cancer Res 2000, 60(6):1541-1545.

10. Shafee N, Kaluz S, Ru N, Stanbridge EJ: PI3K/Akt activity has variable cell-specific effects on expression of HIF target genes, CA9 and VEGF, in human cancer cell lines. Cancer Lett 2009, 282(1):109-115.

11. Mottet D, Dumont V, Deccache Y, Demazy C, Ninane N, Raes M, Michiels C: Regulation of hypoxia-inducible factor-1alpha protein level during hypoxic conditions by the phosphatidylinositol 3-kinase/Akt/glycogen synthase kinase 3beta pathway in HepG2 cells. J Biol Chem 2003, 278(33):31277-31285.

12. O'Brien D, Jacob AG, Qualman SJ, Chandler DS: Advances in pediatric rhabdomyosarcoma characterization and disease model development. Histol Histopathol 2012, 27(1):13-22.

13. Karosas AO: Ewing's sarcoma. Am J Health Syst Pharm 2010, 67(19):1599-1605.

14. Scotlandi K: Targeted therapies in Ewing's sarcoma. Adv Exp Med Biol 2006, 587:13-22.

15. Wachtel M, Schafer BW: Targets for cancer therapy in childhood sarcomas. Cancer Treat Rev 2010, 36(4):318-327.

16. Opel D, Poremba C, Simon T, Debatin KM, Fulda S: Activation of Akt predicts poor outcome in neuroblastoma. Cancer Res 2007, 67(2):735-745

17. Cen L, Hsieh FC, Lin HJ, Chen CS, Qualman SJ, Lin J: PDK-1/AKT pathway as a novel therapeutic target in rhabdomyosarcoma cells using OSU-03012 compound. Br J Cancer 2007, 97(6):785-791.

18. Petricoin EF 3rd, Espina V, Araujo RP, Midura B, Yeung C, Wan X, Eichler GS, Johann DJ Jr, Qualman S, Tsokos M, et al: Phosphoprotein pathway mapping: Akt/mammalian target of rapamycin activation is negatively associated with childhood rhabdomyosarcoma survival. Cancer Res 2007 67(7):3431-3440.

19. Ahmed AA, Sherman AK, Pawel BR: Expression of therapeutic targets in Ewing sarcoma family tumors. Hum Pathol 2012, 43(7):1077-1083.

20. Opel D, Naumann I, Schneider M, Bertele D, Debatin KM, Fulda S: Targeting aberrant PI3K/Akt activation by PI103 restores sensitivity to TRAIL-induced apoptosis in neuroblastoma. Clin Cancer Res 2011, 17(10):3233-3247.

21. Belaiba RS, Bonello S, Zahringer C, Schmidt S, Hess J, Kietzmann T, Gorlach A: Hypoxia up-regulates hypoxia-inducible factor-1alpha transcription by involving phosphatidylinositol 3-kinase and nuclear factor kappaB in pulmonary artery smooth muscle cells. Mol Biol Cell 2007, 18(12):4691-4697.

22. Ardyanto TD, Osaki M, Tokuyasu N, Nagahama Y, Ito $\mathrm{H}$ : CoCl2-induced HIF-1alpha expression correlates with proliferation and apoptosis in MKN-1 cells: a possible role for the PI3K/Akt pathway. Int J Oncol 2006, 29(3):549-555

23. Dekanty A, Lavista-Llanos S, Irisarri M, Oldham S, Wappner P: The insulin$\mathrm{PI3K} / \mathrm{TOR}$ pathway induces a HIF-dependent transcriptional response in Drosophila by promoting nuclear localization of HIF-alpha/Sima. J Cell Sci 2005, 118(Pt 23):5431-5441.

24. Zhou J, Schmid T, Frank R, Brune B: PI3K/Akt is required for heat shock proteins to protect hypoxia-inducible factor 1alpha from $\mathrm{pVHL}$ independent degradation. J Biol Chem 2004, 279(14):13506-13513.

25. Jiang BH, Jiang G, Zheng JZ, Lu Z, Hunter T, Vogt PK: Phosphatidylinositol 3-kinase signaling controls levels of hypoxia-inducible factor 1. Cell Growth Differ 2001, 12(7):363-369.

26. Opel D, Westhoff MA, Bender A, Braun V, Debatin KM, Fulda S: Phosphatidylinositol 3-kinase inhibition broadly sensitizes glioblastoma 
cells to death receptor- and drug-induced apoptosis. Cancer Res 2008, 68(15):6271-6280

27. Bedogni B, Warneke JA, Nickoloff BJ, Giaccia AJ, Powell MB: Notch1 is an effector of Akt and hypoxia in melanoma development. J Clin Invest 2008, 118(11):3660-3670.

28. Burrows N, Babur M, Resch J, Ridsdale S, Mejin M, Rowling EJ, Brabant G, Williams KJ: GDC-0941 inhibits metastatic characteristics of thyroid carcinomas by targeting both the phosphoinositide-3 kinase (PI3K) and hypoxia-inducible factor-1alpha (HIF-1alpha) pathways. J Clin Endocrinol Metab 2011, 96(12):E1934-E1943.

29. DeBerardinis RJ, Lum JJ, Hatzivassiliou G, Thompson CB: The biology of cancer: metabolic reprogramming fuels cell growth and proliferation. Cell Metab 2008, 7(1):11-20.

30. Jiao M, Nan KJ: Activation of PI3 kinase/Akt/HIF-1alpha pathway contributes to hypoxia-induced epithelial-mesenchymal transition and chemoresistance in hepatocellular carcinoma. Int J Oncol 2012, 40(2):461-468.

31. Argyriou P, Papageorgiou SG, Panteleon V, Psyrri A, Bakou V, Pappa V, Spathis A, Economopoulou P, Papageorgiou E, Economopoulos T, et al: Hypoxiainducible factors in mantle cell lymphoma: implication for an activated mTORC1 > HIF-1alpha pathway. Ann Hematol 2011, 90(3):315-322.

32. Zhao Y, Liu H, Riker Al, Fodstad O, Ledoux SP, Wilson GL, Tan M: Emerging metabolic targets in cancer therapy. Front Biosci 2011, 16:1844-1860.

33. Bian CX, Shi Z, Meng Q, Jiang Y, Liu LZ, Jiang BH: P70S6K 1 regulation of angiogenesis through VEGF and HIF-1alpha expression. Biochem Biophys Res Commun 2010, 398(3):395-399.

34. Fang J, Ding M, Yang L, Liu LZ, Jiang BH: PI3K/PTEN/AKT signaling regulates prostate tumor angiogenesis. Cell Signal 2007, 19(12):2487-2497.

35. Fox PL, Mazumder B, Ehrenwald E, Mukhopadhyay CK: Ceruloplasmin and cardiovascular disease. Free Radic Biol Med 2000, 28(12):1735-1744.

36. Liu L, Ning X, Han S, Zhang H, Sun L, Shi Y, Sun S, Guo C, Yin F, Qiao T, et al: [Hypoxia induced HIF-1 accumulation and VEGF expression in gastric epithelial mucosa cell: involvement of ERK1/2 and PI3K/Akt]. Mol Biol (Mosk) 2008, 42(3):459-469.

37. Li L, Qu Y, Mao M, Xiong Y, Mu D: The involvement of phosphoinositid 3-kinase/Akt pathway in the activation of hypoxia-inducible factor-1alpha in the developing rat brain after hypoxia-ischemia. Brain Res 2008, 1197:152-158

38. Li L, Qu Y, Mao M, Mu D: [Phosphoinositide 3-kinase/Akt pathway involved in regulation of hypoxia inducible factor 1alpha in hypoxia ischemia brain damage of neonatal rats]. Zhongguo Xiu Fu Chong Jian Wai Ke Za Zhi 2008, 22(9):1102-1107.

39. Arsham AM, Plas DR, Thompson CB, Simon MC: Phosphatidylinositol 3-kinase/Akt signaling is neither required for hypoxic stabilization of HIF-1 alpha nor sufficient for HIF-1-dependent target gene transcription. J Biol Chem 2002, 277(17):15162-15170.

40. Alvarez-Tejado M, Alfranca A, Aragones J, Vara A, Landazuri MO, del Peso L: Lack of evidence for the involvement of the phosphoinositide 3-kinase/Akt pathway in the activation of hypoxia-inducible factors by low oxygen tension. J Biol Chem 2002, 277(16):13508-13517.

41. Coutte L, Dreyer C, Sablin MP, Faivre S, Raymond E: [PI3K-AKT-mTOR pathway and cancer]. Bull Cancer 2012, 99(2):173-180.

42. Bender A, Opel D, Naumann I, Kappler R, Friedman L, von Schweinitz D, Debatin KM, Fulda S: PI3K inhibitors prime neuroblastoma cells for chemotherapy by shifting the balance towards pro-apoptotic Bcl-2 proteins and enhanced mitochondrial apoptosis. Oncogene 2011, 30(4):494-503.

43. Mukhopadhyay CK, Mazumder B, Fox PL: Role of hypoxia-inducible factor-1 in transcriptional activation of ceruloplasmin by iron deficiency. $J$ Biol Chem 2000, 275(28):21048-21054.

doi:10.1186/1475-2867-13-36

Cite this article as: Kilic-Eren et al:: Targeting PI3K/Akt represses Hypoxia inducible factor $1 a$ activation and sensitizes Rhabdomyosarcoma and Ewing's sarcoma cells for apoptosis. Cancer Cell International 2013 13:36.

\section{Submit your next manuscript to BioMed Central and take full advantage of:}

- Convenient online submission

- Thorough peer review

- No space constraints or color figure charges

- Immediate publication on acceptance

- Inclusion in PubMed, CAS, Scopus and Google Scholar

- Research which is freely available for redistribution

Submit your manuscript at www.biomedcentral.com/submit
() Biomed Central 